\section{Case Reports in Dermatology}

\title{
Bullous Pemphigoid in Which Eruption Developed Exclusively on Preexisting Eruption of Vitiligo Vulgaris
}

\author{
Masahiro Oka ${ }^{a}$ Takeshi Fukumoto ${ }^{b}$ \\ ${ }^{a}$ Department of Dermatology, Kita-Harima Medical Center, Ono City, Japan; ${ }^{b}$ Division of \\ Dermatology, Department of Internal Related, Kobe University Graduate School of \\ Medicine, Kobe, Japan
}

\section{Keywords}

Autoimmune regulator - Bullous pemphigoid - Macrophage migration inhibitory factor .

Vitiligo vulgaris

\begin{abstract}
Coexistence of bullous pemphigoid (BP) and vitiligo vulgaris (VV) is very rare. We present a unique case of BP associated with VV in a 76-year-old Japanese man, in which BP eruption developed exclusively on preexisting $\mathrm{V} V$ regions. The patient was referred to us with a 3-month history of blistering eruption with severe pruritis on the right forearm and left lower limb. In addition, he had been suffering from a widespread depigmented eruption on the trunk and extremities for at least 20 years. Curiously, the blistering eruption developed exclusively on preexisting depigmented eruption. Histopathological and laboratory examinations identified the blistering eruption as BP. The depigmented eruption was diagnosed as VV. BP eruption responded quickly to oral prednisolone therapy, improving within 1 week, and the prednisolone dose was gradually tapered. Within 1 month, BP lesions almost completely resolved. In the present case, BP not only coexisted with $\mathrm{VV}$, but also developed exclusively on preexisting vitiliginous regions. The present case strongly suggests that BP and VV are partly caused by common pathological mechanisms.




\section{Introduction}

Bullous pemphigoid (BP) and vitiligo vulgaris (VV) are both autoimmune skin disorders and are occasionally associated with other autoimmune diseases [1, 2]. However, coexistence of BP and VV is extremely rare [3-5]. Here, we describe the case of a patient with BP associated with VV. Curiously, BP eruption developed exclusively on preexisting VV eruption.

\section{Case Report}

A 76-year-old Japanese man was referred to us with a 3-month history of blistering eruption with severe pruritus on the right forearm and left lower limb. In addition, he had been suffering from a widespread depigmented eruption on the trunk and extremities for at least 20 years. Although this depigmented eruption had been treated with topical ointment (details unknown) when it first developed, the eruption continued to enlarge and had remained almost unchanged since he was at least 60 years old. He had never received phototherapy for the depigmented eruption. Physical examination revealed tense bullae and erosions on the extensor aspects of the right forearm, inner aspect of the left lower limb, extensor sites of the ankles, and inner side of the left foot (Fig. 1a-c). Widespread vitiliginous macules were present on the trunk, extremities, buttocks, and genital region (Fig. 1d). Importantly, all bullae and erosions were present over the vitiliginous macules (Fig. 1a-d). Laboratory tests showed high levels of serum anti-BP180 antibody (148 U/mL; normal, 0-8.9 U/mL). Histopathological examination of a skin biopsy from a bulla on the extensor surface of the left ankle revealed a subepidermal blister containing numerous eosinophils (Fig. 1e). Direct immunofluorescence detected the presence of linear immunoglobulin G (Fig. 1f) and C3 (Fig. 1g) deposits along the subepidermal basal membrane zone. The bullous lesions and depigmented macules were diagnosed as BP and VV, respectively. BP was treated with an initial prednisolone dose of 30 $\mathrm{mg} /$ day. The bullous eruption responded quickly to therapy, and the prednisolone dose was gradually tapered. Within 1 month, BP lesions had disappeared almost completely.

\section{Discussion/Conclusion}

Although BP and VV coexisted in the present case, determining whether the coexistence of the two diseases was coincidental or represented a true association is difficult, since only 3 other cases [3-5] of coexisting BP and VV have been described. A large population study on the prevalence of comorbidities among $\mathrm{BP}$ or $\mathrm{VV}$ is required to examine whether these two diseases can be significantly associated.

In the present case, BP not only coexisted with $\mathrm{VV}$, but also developed exclusively on preexisting vitiliginous regions. In all 3 previously reported cases of coexisting BP and VV [35], although detailed positional relationships between BP eruption and VV eruption were not described, the BP eruption seems to have developed at least on skin without VV. The phenomenon of BP eruption developing only on preexisting VV lesions in the present case is thus intriguing. Although we cannot exclude the possibility that this phenomenon was purely coincidental, the fact that more than $10 \mathrm{BP}$ lesions were all superimposed on VV lesions strongly suggests that this phenomenon was more than fortuitous. Interestingly, in the previously reported case of concomitant psoriasis vulgaris, VV, and BP [5], BP lesions preferentially developed on preexisting psoriatic plaques in a specific area. Further investigation is necessary to 
elucidate the mechanism by which BP eruption develops preferentially on areas already affected by other autoimmune skin diseases.

In conclusion, the present case, together with the previous reports of coexistence of BP and $\mathrm{VV}$, suggests a common pathological pathway between the two diseases.

\section{Statement of Ethics}

Informed consent was obtained from the patient. The study complied with the Declaration of Helsinki.

\section{Disclosure Statement}

The authors declare no conflict of interest.

\section{Funding Sources}

None of the authors received any financial support for the present study.

\section{Author Contributions}

All authors have contributed significantly. Dr. Oka conducted the dermatological examinations of the patient. In addition, Dr. Oka wrote the manuscript. Dr. Fukumoto performed the histological analyses. All authors agree with the content of the manuscript.

\section{References}

1 Callen JP. Internal disorders associated with bullous disease of the skin. A critical review. J Am Acad Dermatol. 1980 Aug;3(2):107-19.

2 Iannella G, Greco A, Didona D, Didona B, Granata G, Manno A, et al. Vitiligo: Pathogenesis, clinical variants and treatment approaches. Autoimmun Rev. 2016 Apr;15(4):335-43.

3 Hamilton DV, McKenzie AW. Bullous pemphigoid and primary biliary cirrhosis. Br J Dermatol. 1978 Oct;99(4):447-50.

4 Pasić A, Ljubojević S, Lipozencić J, Marinović B, Loncarić D. Coexistence of psoriasis vulgaris, bullous pemphigoid and vitiligo: a case report. J Eur Acad Dermatol Venereol. 2002 Jul;16(4):426-7.

5 Jankowski M, Czajkowski R, Scibior K, Schwartz RA. Coexistence of psoriasis vulgaris and vitiligo with bullous pemphigoid: a case report. Int J Dermatol. 2014 Jul;53(7):e359-61. 


\section{Case Reports in Dermatology}
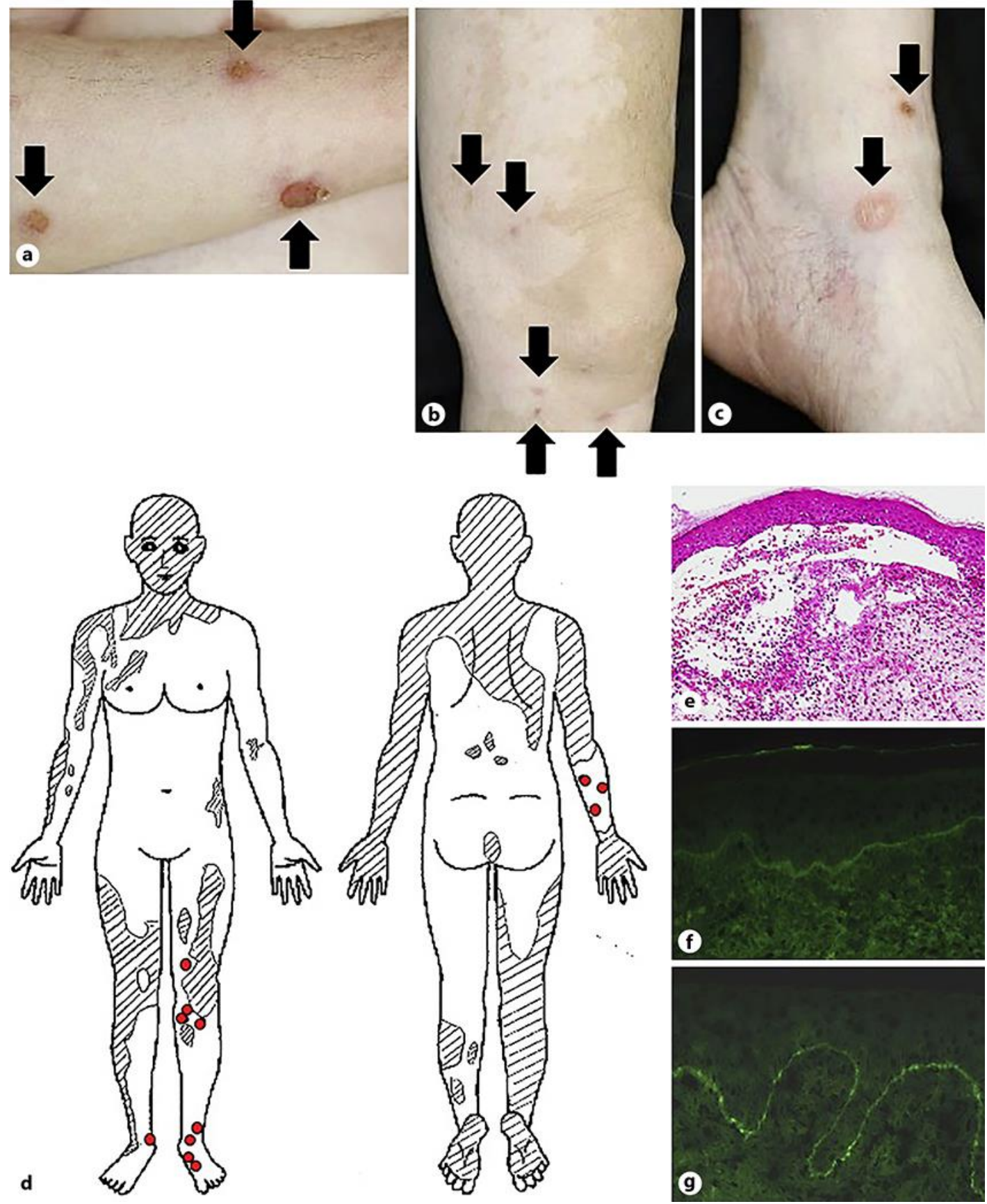

Fig. 1. a-c Clinical appearance of the skin lesions. Erosions and tense bullae (arrows) over vitiliginous macules on the extensor aspect of the right forearm (a), inner aspect of the left lower extremity (b), and extensor site of the left ankle (c). $\mathbf{d}$ The distribution of skin lesions (red circles), depigmented lesions (white areas), and intact skin areas (areas with oblique lines). e Histopathological findings of a bulla on the extensor site of the left ankle. A subepidermal blister containing numerous eosinophils (hematoxylin and eosin, original magnification $\times 200$ ). $\mathbf{f}, \mathbf{g}$ Direct immunofluorescence findings. Linear immunoglobulin G (f) (original magnification $\times 400$ ) and C3 $(g)$ (original magnification $\times 400$ ) deposition at the dermal-epidermal junction. 